ANUARIO DE Estudios MEdIEVALES

47/1, enero-junio de 2017, pp. 211-237

ISSN 0066-5061

doi:10.3989/aem.2017.47.1.08

\title{
LA DIFFUSION EN EUROPE DE LA NOUVELLE DE L'OCCUPATION DE DAMAS EN 802/1400-803/1401 PAR TAMERLAN ET SES CONSÉQUENCES SUR LE COMMERCE MÉDITERRANÉEN
}

\author{
THE CIRCULATION IN EUROPE OF THE NEWS OF THE \\ OCCUPATION OF DAMASCUS IN 802/1400-803/1401 \\ BY TAMERLANE AND ITS CONSEQUENCES \\ FOR MEDITERRANEAN TRADE
}

PIERRE MOUKARZEL Université Libanaise http://orcid.org/ 0000-0002-7081-0151

\begin{abstract}
Résumé: En 802/1400-803/1401, Tamerlan occupa Damas. Les marchands européens partirent de la Syrie et la nouvelle de cet événement ne tarda pas à circuler dans les principaux centres commerciaux européens semant la panique en perturbant le fonctionnement des marchés et bouleversant les prix des épices. Par quels moyens cette nouvelle fut-elle diffusée et quelles furent ses séquelles sur l'ensemble du commerce méditerranéen?

Mots clés: Tamerlan; Damas; circulation des nouvelles; Venise; compagnie Datini; épices; concurrence; spéculations.
\end{abstract}

Abstract: In 802/1400-803/1401 Tamerlane occupied Damascus. European merchants abandoned Syria and the news of this event was soon circulating around the main European trading centres, spreading panic by disrupting the functioning of markets and upsetting the prices of spices. By what means was this news spread and what were its consequences for Mediterranean trade as a whole?

Keywords: Tamerlane; Damascus; circulation of news ; Venice; Datini company; spices; competition; speculations.

\section{SOMMAIRE}

1. Introduction.- 2. Venise: le centre de la diffusion des nouvelles en Europe.- 3. La vitesse de la transmission de la nouvelle. 4.- Les répercussions de la nouvelle sur les marchés des épices en Europe. 5.- Conclusion. 6.- Bibliographie. 


\section{INTRODUCTION}

À la fin du Moyen Âge, aux XIVee et XVe siècles, les échanges commerciaux entre l'Europe et le Levant connurent un grand essor. La technique commerciale (la lettre de change, le chèque, la comptabilité, l'assurance maritime) se développa de manière remarquable, de même que les facilités et les techniques des transports maritimes. De nouvelles techniques furent adoptées dans la construction des navires effectuant de longs voyages. Pour le commerce des épices, les Vénitiens utilisèrent des galères qui pouvaient porter une charge d'environ 200 à 300 tonnes métriques ${ }^{1}$. Les coques génoises portaient 1000 tonnes métriques ${ }^{2}$. L'invention de la boussole à pivot, des portulans et d'autres instruments facilita la navigation : en Méditerranée, le navire vainquit l'hiver ${ }^{3}$. Venise, par exemple, ne ferma plus la mer en novembre à ses convois du Levant. Tout cela aboutit à une intensification des échanges à travers la Méditerranée et à un changement considérable dans l'implantation et la fixation des marchands occidentaux avec le Levant : une classe marchande très riche fut alors formée ${ }^{4}$.

Les marchands qui avaient leurs activités commerciales entre le Levant et l'Europe avaient besoin d'informations courantes sur les fluctuations dans les échanges commerciaux. Dans le but de savoir ce qu'ils devaient vendre ou garder en stock, ils se renseignaient sur le développement des prix des épices en Orient, en raison de la concurrence commerciale entre les différentes villes marchandes méditerranéennes ${ }^{5}$. De même, les marchands qui résidaient dans leurs pays et dirigeaient leurs affaires à partir de leurs sièges centraux désiraient savoir régulièrement les variations de prix, de mise en vente, de demande des différents produits avant d'expédier des marchandises sur des navires en partance vers le Levant. Les lettres conservées dans les archives de la compagnie de Datini de Prato et des compagnies vénitiennes du $\mathrm{XV}$ siècle montrèrent qu'il existait des réseaux d'informations efficaces, fournissant régulièrement des nouvelles et tissant des liens continus entre les marchands d'Europe et ceux du Levant. ${ }^{6}$ Ainsi ces lettres peuvent être considérées comme l'une des rares sources susceptibles de nous transmettre une masse importante de nouvelles sous leur forme originelle. Elles constituent une mine de renseignements de toute espèce, événements politiques et rumeurs, transac-

\footnotetext{
${ }^{1}$ Hocquet 1979, p. 91.

${ }^{2}$ Melis 1984, p. 14.

${ }^{3}$ Lane 1963, pp. 605-617; Tucci 1958, pp. 72-86.

${ }^{4}$ Ashtor 1983, p. 367.

${ }^{5}$ Sardella 1948, p. 31.

${ }^{6}$ Congdon 1997, pp. 157-171.
} 
tions commerciales, données biographiques et épisodes de la vie quotidienne, témoignages sur la variété de la langue vernaculaire, etc ${ }^{7}$.

Les marchands, dans toute la région méditerranéenne et pendant la période du bas Moyen Âge, avaient constamment besoin de nouvelles. Ils cherchaient les informations concernant les lieux des marchés, les marchandises, et la façon de participer au commerce sans subir des contraintes de la part de l'administration locale et de la population indigène. Ils devaient choisir le moment convenable pour investir leurs capitaux ou suspendre leurs activités dans une région donnée à cause d'un trouble interne, déterminer comment et quand profiter d'une brève période de paix, et prendre les précautions contre les pertes au cas où les trajets et les itinéraires qu'empruntaient leurs marchandises étaient risqués. Les marchands s'intéressèrent aux événements dans une région qui pouvaient affecter une autre, et quand reprendre les affaires lorsque les conditions redevenaient normales. Les informations les plus importantes pour les marchands étaient les changements et les variations des conditions pour le bon déroulement des affaires: un marché pouvait soudainement fermer ou s'ouvrir, tandis que les investissements qui avaient semblé être sûrs pouvaient soudainement, comme par un retour de balancier, devenir des désastres épouvantables. La vitesse de réception des nouvelles et le temps de réaction étaient cruciaux et pouvaient être déterminants pour le succès ou la faillite. Les nouvelles étaient la pierre angulaire de la prospérité ou de la chute d'un marchand ${ }^{8}$.

Pour la fin du XIVe siècle, les documents conservés dans les archives de la compagnie florentine de Francesco di Marco Datini ont fourni une masse fantastique d'informations et une grande diversité d'acteurs commerciaux et de correspondants. Ce fonds d'archives produit par le réseau d'une agence d'envergure moyenne et les relations personnelles d'un homme riche mais sans relief politique, contient un grand nombre de lettres concentrées sur la période 1380-1410. Federigo Melis a compté plus de 152.000 lettres individuelles et autour de 500 livres de compte dans ces archives ${ }^{9}$. Aucune collection comparable pour un marchand, retraçant la grande majorité de sa carrière et des activités marchandes, n'a existé pendant les périodes médiévales ou au début des périodes modernes. Ces archives sont une partie des rapports produits par sept sociétés de Datini et de nombreux correspondants. Cependant, la perte de nombreux registres d'archives est ici très regrettable vu que, parmi plus de 6.000 articles reçus de Venise par Francesco Di Marco Datini et ses

\footnotetext{
${ }^{7}$ Hayez 1994, p. 64. Pour plus d'informations sur l'analyse du contenu et du lexique des lettres marchandes, voir: Hayez 2004, pp. 113-134.

${ }^{8}$ Favier 1987, p. 83.

${ }^{9}$ Melis 1962, pp. 30-32.
} 
compagnies, uniquement deux douzaines de lettres écrites à cette ville par Datini ou par ses agents ont été conservées. Toutefois, en comparaison avec d'autres archives groupant des documents en rapport avec les marchands, les archives de Datini sont très complètes. La plupart des événements historiques qui pouvaient influencer les activités commerciales sont mentionnés par un ou plusieurs correspondants au cours de ces échanges épistolaires.

En 802/1400-803/1401, la Syrie ${ }^{10}$ fut occupée par Tamerlan (Tīmūr Lank) et la ville de Damas fut détruite en grande partie ${ }^{11}$. Damas, dès l'époque omeyyade, fut toujours considérée comme le centre du pouvoir de la Syrie du Sud. En outre, elle était le principal centre commercial en Syrie durant le Moyen Âge attirant les marchands de différentes nationalités et où arrivaient les caravanes chargées d'épices et de soie de la Mecque, d'Asie Centrale, de Bagdad, de Sultanieh et de Tabriz ${ }^{12}$. En prenant cet événement comme exemple, nous allons essayer de retracer le cadre dans lequel s'effectua la circulation des nouvelles entre le Levant et l'Europe par voie maritime : les moyens de transport, les itinéraires et les durées des trajets, l'impact des nouvelles provenant de l'Orient sur le réseau commercial méditerranéen ${ }^{13}$.

D'après les lettres conservées dans les archives Datini, portant sur le sujet des nouvelles de l'avancée de Tamerlan et de l'occupation de Damas qui furent diffusées dans les principales villes marchandes européennes les faits sont cités comme suit : le 18 décembre, un navire partit de Beyrouth et arriva à Candie (île de Crète) rapportant une information très importante : ...Tamerlan incendia Damas excepté le château... ${ }^{14}$. Le 23 janvier, un autre navire partit

\footnotetext{
${ }^{10}$ L'utilisation du mot "Syrie" dans l'article nécessite une définition. Par "Syrie", nous entendons un espace géographique que les Arabes appellent Bilād al-S̄ām et qui englobe les États actuels : la Syrie, le Liban, la Palestine, et la Jordanie.

${ }^{11}$ Voir sur ce sujet: Ibn Tag̉rī Birdī 1992, vol. XII, pp. 175-195; Ibn Tag̉nī Birdī 1986 , pp. 118-125; Al-Maqrīzī 1997, vol. VI, pp. 50-54; Ibn Hağar 1969-1972, vol. II, pp. 133-140.

${ }^{12}$ Les caravanes qui partent de Damas vers la Mecque sont celles des pèlerins musulmans qui visitent la Mecque et les lieux saints une fois par an, et chargent au retour les épices. Le pèlerinage à la Mecque varie chaque année et tombe dans toutes les saisons, mais il semble que le commerce des épices en provenance de la Mecque s'effectue durant une certaine période qui coïncide avec l'arrivée des navires occidentaux, en particulier les galères vénitiennes, au port de Beyrouth en été et en automne. Il s'agit de la muda de Beyrouth. Stöckly 1995, pp. 143-152.

${ }^{13}$ Il faut signaler que les lettres conservées par la compagnie Datini de Prato traitant la circulation des nouvelles de l'occupation de Damas par Tamerlan furent mentionnées et exposées par Federigo Melis mais sans une analyse et une exploitation suffisantes de leur contenu. Il se limita tout simplement à énumérer des lettres, mais une étude de l'impact des nouvelles de Tamerlan en Syrie sur le commerce méditerranéen manque. En outre, il n'a pas expliqué comment la destruction de Damas et la diffusion des nouvelles ont modifié les caractères du trafic des principales villes marchandes européennes avec le Levant.

${ }^{14}$ C'est la nef de deux marchands de Barcelone, Guillem Pasadores et Francesc Fogassot, qui rapporta la nouvelle de l'invasion de Damas par Tamerlan aux facteurs de la compagnie Datini à Candie. Coulon 2004, p. 366.
} 
de Candie en direction de Venise. De là, les nouvelles furent transmises à la compagnie florentine des Ricci à Gênes qui les partagea avec la compagnie Datini dans la même ville. De Gênes, la compagnie Datini envoya les nouvelles à l'autre branche de la compagnie à Barcelone, puis les membres de la compagnie Datini à Gênes reçurent les nouvelles avec prudence et méfiance et les transmirent à leurs collègues à Barcelone en écrivant : pourtant il ne faut pas y croire parce que les Vénitiens pourraient faire ceci pour faire monter les prix de leurs épices. Nous saurons la vérité et nous vous aviserons de ce que nous entendrons ${ }^{15}$. De leur côté, les agents de la compagnie Datini à Barcelone transmirent les nouvelles à la filiale de Majorque et signalèrent qu'ils avaient reçu deux lettres : une en provenance de Gênes et l'autre de Florence. De plus, le 20 janvier 1401, l'association commerciale (la Comissaria) de Zanobi Gaddi et d'Antonio di ser Bartolomeo représentant la compagnie Datini, envoya de son siège à Venise un messager à Barcelone muni d'une lettre contenant le sujet de la prise de Damas par Tamerlan- la nouvelle venait par le biais du coassocié Antonio qui arriva à Venise, retournant de Jérusalem, le 9 janvier. Ainsi, les nouvelles empruntèrent de différents trajets pour arriver à Barcelone : Beyrouth-Barcelone ${ }^{16}$, Beyrouth-Candie-Venise-Gênes-Barcelone, BeyrouthCandie-Venise-Florence-Barcelone, et Jérusalem-Venise-Barcelone ${ }^{17}$. Le 20 février 1401, la même association commerciale de Zanobi Gaddi et d'Antonio di ser Bartolomeo, de son siège à Gênes, transmit les nouvelles de l'invasion de Tamerlan à Valence et à Majorque, et encore même, le 25 février, à Florence d'où elles furent renvoyées, à l'initiative d'une autre compagnie, à Gênes annonçant la variation des prix des principaux produits orientaux, des épices et du $\operatorname{coton}^{18}$. Les nouvelles provenant du Levant ne tardèrent pas à circuler dans les centres commerciaux européens : une lettre écrite à Alexandrie le 13 février prit la direction de Candie et arriva à Venise le 21 mars où elle fut réexpédiée à Barcelone le 3 mai ${ }^{19}$; les nouvelles arrivèrent à Gaète en février ; le $1^{\text {er }}$ mai, un

15 “Ècci da Vinegia lettera ne' Ricci e dichono v'era nuove di Candia, per una nave, partì a dì 23 di gennaio di là, chome v'era venuto una nave de Baruti, partì a dì 18 dicenbre, e contava Tanburlano avìa arso Domascho, tutto, salvo il castello. Non vi si dà, però, piena fede: perché i viniziani fan(n)o ciò possono per far montare le loro spezie. Sapras(s)i il vero: e quel sentiremo v'aviseremo". Datini, n. 881, lett. Genova-Barcellona, Comp. Datini, 20. 2. 1401, c. 2, citée par Melis 1962, p. 30.

${ }^{16}$ La nouvelle arriva d'abord directement à Barcelone avec le premier navire barcelonais parti de Beyrouth.

${ }^{17}$ Datini, n. 1053, lett. Barcellona-Maiorca, Comp. Datini, 12 .3. 1401; Datini, n. 927, lett. Venezia-Barcellona, Comissaria di Zanobi Gaddi e Antonio di ser Bartolomeo, 20. 1. 1401, Melis 1962, pp. 30-31.

${ }^{18}$ Datini, n. 993, lett. Genova-Valenza, Comp. Datini, 20. 2. 1401, c .2; Datini, n. 1071, lett Genova-Maiorca, Comp. Datini, 20.2.1401; Datini, n. 662, lett. Genova-Firenze, Comp. Datini, 25.2.1401, ibidem, p. 31.

${ }^{19}$ Ainaud 1965, doc. 1, pp. 332-333. 
navire de Collioure rentrant d'Alexandrie apporta à Ibiza les nouvelles des mouvements de l'armée de Tamerlan après avoir quitté Damas en direction de l'Égypte et un autre navire venant de Rhodes les transmit à la compagnie Tecchini de Perpignan puis l'information circula de Rome à Barcelone le 28 juin $^{20}$.

D'autre part, les registres du Sénat vénitien conservés aux archives d'État de Venise fournissent des informations sur la circulation des nouvelles des conquêtes de Tamerlan par d'autres canaux de transmission: $\mathrm{Pa}-$ olo Zane, le consul vénitien à Damas, réfugié à Famagouste, envoya une lettre qui arriva à Venise le 11 novembre 1400 donnant des nouvelles de la Syrie et de la situation à Damas ${ }^{21}$.

De tout ce qui se précède, nous pouvons en déduire la synthèse qui suit.

\section{VENISE: LE CENTRE DE LA DIFFUSION DES NOUVELLES EN EUROPE}

Le contenu des lettres des archives de Datini montre que si un marchand voulait des informations sur le Levant, il serait bien servi en ayant un correspondant à Venise. Elle fut une des villes marchandes les plus importantes à la fin du XIVe siècle où arrivaient les nouvelles des événements de partout, surtout ceux qui pouvaient affecter les marchands et leurs activités et menacer les échanges commerciaux. À cause des intérêts commerciaux de Venise si répandus, les marchands vénitiens étaient les premiers à être tenus au courant des événements qui se déroulaient dans les ports et les villes de la Méditerranée où ils étaient présents et/ou représentés; ils notaient dans leurs rapports tout ce qu'ils entendaient ou voyaient durant leur séjour à l'étranger. Les marchands de Venise avaient une gamme plus large d'informations que les marchands d'autres lieux ; ils les rassemblaient et les disséminaient ensuite par leur correspondance. En outre, les autres réseaux marchands européens à travers la Méditerranée collectaient également les informations, les recueillaient et les envoyaient, tant aux autres marchands dans les grands centres de redistribution de l'information, comme Venise, qu'aux lieux où elles pouvaient être intéressantes pour le déroulement du commerce.

À la fin du Moyen Âge, Venise organisa des réseaux maritimes en Méditerranée, en mer Noire et en mer du Nord, permettant de faire circuler

${ }^{20}$ Datini, n. 1072, lett. Iviza-Maiorca, Tucci di Gennaio, 29. 4(1. 5). 1401, c. 2t; Datini, n. 777, lett. Gaeta-Genova, Comp.d'Agnolo di ser Pino e Giuliano di Giovanni Portinari, 22. 2. 1401; Datini, n. 911, lett. Roma-Barcellona, Comp.d'Agnolo di ser Pino e Giuliano di Giovanni Portinari, 28.6.1401, citées par ibidem, p. 32.

${ }^{21}$ Archivio di Stato di Venezia, Senato Misti, reg. 45, f. 49 r ${ }^{\circ}$. 
biens, nouvelles, informations et hommes. Ils rythmèrent l'activité commerciale de Venise et celle d'autres villes marchandes. La navigation vénitienne était composée en grande partie de convois de galères et de coques (les mude) sous la gestion directe du Sénat vénitien, mais il existait aussi une navigation libre et désarmée qui joua un rôle important dans le transport maritime de Venise $^{22}$ : la navigation commerciale vénitienne se répartissait donc entre deux secteurs d'activité complémentaires. Les galères publiques et les nefs privées assuraient l'approvisionnement de Venise en épices comme en matières premières. La gestion des deux organisations supposait des choix économiques et politiques significatifs de la part des acteurs ${ }^{23}$. Les navires privés, que les autorités vénitiennes ne règlementaient guère, effectuaient sans doute le gros du transport maritime vénitien, en partie illicitement, faisant voile vers les différents ports de la Méditerranée, dont Beyrouth et Alexandrie ${ }^{24}$.

Là il serait bon d'indiquer que l'organisation des convois maritimes n'était pas une exclusivité de Venise. Gênes et Barcelone jouaient également un rôle principal dans les échanges commerciaux internationaux sur une grande échelle en Europe à la fin du Moyen Âge. Concernant le commerce avec le sultanat mamelouk, Gênes avait un convoi de galères organisé par l'État appelé galere di Siria composé de deux galères qui touchaient aussi Chypre. Ces galères ne partaient pas de Gênes à une date fixe, elles effectuaient le voyage au printemps et parfois au début de l'automne. En outre, des navires génois fréquentaient Alexandrie ${ }^{25}$. De Barcelone, les premiers convois réguliers des galères vers l'Égypte et la Syrie furent organisés dès 1394, mais ces départs groupés ne durèrent que jusqu'en 1397. Le trafic catalan avec le sultanat mamelouk s'intensifia, et la moyenne des départs vers l'Égypte et la Syrie atteignit ainsi les huit navires entre 1395 et 1401 et quatorze navires entre 1402 et $1405^{26}$. Mais c'est à Venise que l'organisation des convois maritimes atteignit un haut degré de perfection et de spécialisation, d'autant que Venise put maintenir un service de galères et de coques avec l'Égypte et la Syrie tout au long du règne mamelouk. Les réseaux maritimes vénitiens furent les plus diversifiés, les plus réguliers et les plus efficaces, non seulement en Méditerranée, mais aussi en mer Noire et en mer du Nord à la fin du Moyen Âge; ils se distinguaient par plusieurs qualités: la vitesse, le rythme, la fréquence et l'intensité.

${ }^{22}$ Luzzatto 1954, pp. 53-57. Voir aussi Doumerc 1911, pp. 357-395; Hocquet 1991, pp. 397-434; Christ 2011, pp. 55-65. Il faut signaler que les lignes de service maritime organisées par l'État vénitien se répartissaient sur sept lignes: celles de Romanie/mer Noire, de Chypre/Arménie, de Flandre, d'Alexandrie, de Beyrouth, d'Aigues-Mortes et de Barbarie.

${ }_{23}^{23}$ Judde de Larivière 2008, pp. 60-61.

${ }^{24}$ Christ 2010, p. 279.

${ }^{25}$ Musso 1975, pp. 73-74.

${ }^{26}$ Coulon 2004, pp. 196-197. 


\section{LA VITESSE DE LA TRANSMISSION DE LA NOUVELLE}

Les lettres circulaient par voie terrestre et par voie maritime, visant à consolider les rapports et le contact entre les marchands de la même nation, dans les villes où ils étaient installés et dans leurs pays. La transmission des lettres entre les principales villes syriennes et l'Égypte variaient et les délais pouvaient s'étendre à plusieurs jours. Les marchands vénitiens de Syrie profitaient du transport de marchandises sur des mulets ou des dromadaires pour expédier des lettres ou en recevoir ${ }^{27}$. Ils y confiaient leur courrier et leurs marchandises à des musulmans selon l'habitude adoptée en Orient depuis l'époque fatimide ${ }^{28}$. Mais les nouvelles les plus importantes nécessitaient l'envoi d'un courrier direct et individuel. Les personnes engagées pour acheminer les lettres et rien d'autre étaient des professionnels. Les marchands vénitiens utilisaient les courriers à pied qui n'avaient d'autre charge que leurs lettres, qui avançaient à une vitesse bien différente des porteurs de marchandises. Les courriers circulaient, à pied, à un rythme soutenu approchant $65 \mathrm{~km}$ par jour. Un courrier partant d'Alexandrie devait mettre dix à douze jours pour rejoindre Damas et dix jours pour arriver à Acre. Il lui fallait deux jours pour rejoindre Beyrouth au départ d'Acre ${ }^{29}$.

Ainsi l'échange de lettres d'affaires parmi les marchands assura un moyen de contact permanent entre les marchands résidant à l'étranger (marchands voyageurs) et ceux qui étaient dans leurs pays (marchands sédentaires), afin d'être tenus au courant des fluctuations des prix des marchandises dans les marchés, et des événements qui pouvaient affecter leurs intérêts. La circulation des nouvelles n'intéressait pas seulement les marchands mais aussi l'État : tous les consuls en poste à l'étranger devaient transmettre des rapports sur la situation politique et les conditions du marché $^{30}$. Les gouvernements des villes marchandes avaient leurs propres services pour la transmission des courriers. À Venise, il y avait des navires, petits et rapides, appelés grippo qui portaient les nouvelles de l'étranger en une période de temps relativement courte ${ }^{31}$. Les marchands, eux, attendaient le départ et l'arrivée des vaisseaux de commerce pour envoyer et recevoir

\footnotetext{
${ }^{27}$ Vallet 1999, pp. 165-166. Parfois on expédiait les lettres avec les marchandises chargées sur le mulet conduit par les mocari (transporteurs) et parfois chargées sur des dromadaires pour un moindre coût et de meilleures conditions de transports, ibidem, pp. 167-171.

${ }^{28}$ Goiten 1999, p. 285.

${ }^{29}$ Bauden 2010, pp. 185-186.

${ }^{30}$ Doumerc 1994, p. 99.

${ }^{31}$ Ashtor 1983,p. 380. Ces bateaux classiques à voile latine étaient capables de tenir la mer aussi bien que les galères et les coques, et adoptaient également les nouvelles techniques navales comme le gouvernail d'étambot. Ils voyageaient avec peu d'étapes et avec des conditions
} 
des lettres. Mais le délai d'acheminement des nouvelles était parfois lent à cause de la vitesse très limitée des transports: les lettres d'affaires en disaient long sur les plaintes concordantes des marchands à cause de la lenteur du délai d'acheminement des nouvelles ${ }^{32}$. Les délais d'acheminement du courrier étaient très variables. L'étude des itinéraires et des durées de voyage était faite à travers la circulation des lettres parce qu'elles étaient datées (alors qu'on ignorait les durées de voyage pour les marchandises). Tout dépendait du type de navire et de la fréquence du vent favorable à la navigation $^{33}$. Les études faites sur la vitesse du transport maritime en Méditerranée montrent que les durées des voyages n'étaient pas très longues: de Beyrouth et d'Alexandrie à Venise, la durée minimale du voyage ne dépassa pas 30 jours, et la durée maximale varia entre 97 et 112 jours: la durée moyenne fut de 38 à 39 jours $^{34}$. Les durées inférieures ou égales à 40 jours étaient assez fréquentes, même pour des villes lointaines comme Barcelone et Bruges sur l'Atlantique. La vitesse moyenne de tous les navires variait entre 20 et 100 milles/jour, elle dépendait des conditions climatiques et de la direction du vent favorable à la navigation. Les études faites sur le climat de la région de la Méditerranée orientale montrent que les vents d'Est favorables à la navigation vers l'Ouest soufflaient durant la période de mi-octobre à mimai ${ }^{35}$. De ce fait les lettres envoyées de la côte syrienne, ou du bassin méditerranéen oriental, vers l'Europe, au printemps (avril-mai) et en automne (septembre-octobre), prenaient moins du temps que celles envoyées en hiver et au milieu de l'été (juin-juillet-août). Les conditions météorologiques dictaient le rythme de la navigation.

D'après les informations relatives à l'invasion de Tamerlan mentionnées dans les lettres échangées en 802/1400-803/1401 comprenant les dates d'envoi et de réception des lettres, la durée de transmission de nouvelles pourrait être comme suit:

météorologiques favorables pouvaient voyager très vite. Christ 2010, pp. 281-282; Christ 2011, pp. 58-59.

${ }^{32}$ Doumerc 1994, p. 102. Parfois la transmission des lettres, pour gagner du temps, se fait en mer d'un navire à l'autre en utilisant une petite barque appelée copano. Casola 1907, p. 301. Casola était sur une galère de pèlerins et le capitaine reçut des lettres d'une galère de Beyrouth contenant des nouvelles de Venise.

${ }^{33}$ Christ 2011, p. 61.

${ }^{34}$ Melis 1984, p. 210. D'après l'analyse des lettres marchandes faite par Christ, le temps moyen pour la liaison Venise-Alexandrie était de neuf semaines et demie, et une moyenne d'environ huit semaines et demie en sens inverse. Christ 2011, p. 61.

${ }^{35}$ Pryor 2000, p. 3. 
Selon la compagnie florentine des Ricci à Gênes:

\begin{tabular}{|l|c|c|}
\hline \multicolumn{1}{|c|}{ TRAJET } & $\begin{array}{c}\text { DATE DE L'ENVOI } \\
\text { DE LA LETTRE }\end{array}$ & $\begin{array}{c}\text { DATE DE LA RÉCEPTION } \\
\text { DE LA LETTRE }\end{array}$ \\
\hline Candie-Venise & 23 janvier 1401 & $?$ \\
\hline Venise-Gênes & $?$ & $?$ \\
\hline Gênes-Barcelone $^{36}$ & 11 février 1401 & 14 mars 1401 \\
\hline
\end{tabular}

Selon la lettre envoyée de la compagnie Datini de Barcelone à sa filiale de Majorque, la nouvelle circula suivant un autre trajet selon différentes dates :

\begin{tabular}{|l|c|c|}
\hline \multicolumn{1}{|c|}{ TRAJET } & $\begin{array}{c}\text { DATE DE L'ENVOI } \\
\text { DE LA LETTRE }\end{array}$ & $\begin{array}{c}\text { DATE DE LA RÉCEPTION } \\
\text { DE LA LETTRE }\end{array}$ \\
\hline Beyrouth-Candie & 13 décembre 1400 & 8 janvier 1401 \\
\hline Candie-Venise & $?$ & $?$ \\
\hline Venise-Gênes & $?$ & $?$ mars 1401 \\
\hline Gênes-Barcelone $^{37}$ & 2 février 1401 & 24 février 1401 \\
\hline Florence-Barcelone $^{38}$ & 22 janvier 1401 & 15 mars 1401 \\
\hline Barcelone-Majorque $^{2}$ & 12 mars 1401 & \\
\hline
\end{tabular}

De Venise et de Gênes, les deux principales villes marchandes européennes, la diffusion de la nouvelle s'accéléra à partir du mois de février:

\begin{tabular}{|l|c|}
\hline \multicolumn{1}{|c|}{ TRAJET } & DATE DE L'ENVOI DES LETTRES \\
\hline Venise-Barcelone & 20 janvier 1401 \\
\hline Gênes-Valence & 20 février 1401 \\
\hline Gênes-Majorque & 20 février 1401 \\
\hline Gaète-Gênes & 22 février 1401 \\
\hline Gênes-Florence & 25 février 1401 \\
\hline Montpellier-Gênes & 25 avril 1401 \\
\hline
\end{tabular}

36 Datini, n. 881.21, lett. Genova-Barcellona, Ricci Ardingo di Gucciozo e Comp a Datini Francesco di Marco e Comp, 11.2.1401, http://datini.archiviodistato.prato.it/www/query.html [consultation: 06/05/2014].

37 Datini, n. 865.14, lett. Datini Francesco di Marco e Domenico di Cambio a Datini Francesco di Marco e Comp, 22.1.1401, http://datini.archiviodistato.prato.it/www/query.html [consultation: 06/05/2014]

38 Datini, n. 1052, 1053 lett. Barcellona-Maiorca, Tanso Guglielmo a Datini Francesco di Marco e Carocci Cristofano di Bartolo e Comp, 12.3. 1401, http://datini.archiviodistato.prato. it/www/query.html [consultation: 06/05/2014]. 


\begin{tabular}{|l|r|}
\hline Ibiza-Majorque & 1 mai 1401 \\
\hline Montpellier-Gênes & 13 mai 1401 \\
\hline Rome-Barcelone & 28 juin 1401 \\
\hline Perpignan-Barcelone & 10 juillet 1401 \\
\hline
\end{tabular}

Nous remarquons que la nouvelle de l'invasion de la Syrie par l'armée de Tamerlan arriva en Europe à partir d'une série de lettres envoyées vers la fin du mois de janvier au sein du réseau Datini et l'échange de missives contenant les nouvelles récentes arrivées par l'intermédiaire des navires retournant d'Alexandrie, de Rhodes et de Candie mit 7 mois. Entre janvier et février, elle fut diffusée dans les principales villes marchandes, comme Venise, Gênes, Florence et Barcelone, qui possédaient de grandes compagnies commerciales organisées avec des filiales, disposant d'un réseau dense de correspondants et de comptoirs installés dans les villes européennes. Entre avril et juillet, la nouvelle parvint aux villes marchandes du Sud de la France et de l'Espagne, couvrant ainsi la majorité des centres commerciaux européens.

Des nouvelles se rapportant à Tamerlan ont bien été rapportées à travers l'Occident par les itinéraires indiqués, mais d'après les registres du Sénat vénitien, la nouvelle de l'invasion de la Syrie par Tamerlan et l'avance de son armée vers Damas arriva en Europe dans des délais plus courts. Elle atteignit Venise le 11 novembre 1400, environ deux mois avant qu'elle soit diffusée par le réseau commercial de Datini et arriva à Venise en janvier 1401. Il est donc évident que cette nouvelle s'est transmise jusqu'à Venise et à d'autres principales villes commerciales plus rapidement et surtout plus directement que les trajets empruntés pour la transmission des lettres marchandes suivant des itinéraires complexes via Venise, Gênes, Florence et Barcelone: le courrier officiel circulait plus vite que les lettres marchandes. En outre, la nef barcelonaise qui rapporta la nouvelle de l'invasion de Damas par Tamerlan aux facteurs de Datini à Candie venait de quitter Beyrouth le 18 décembre 1400. Suivant les routes empruntées par les navires catalans effectuant des voyages entre Barcelone et le sultanat mamelouk après les années 1380, les escales commerciales régulièrement mentionnées par les sources notariées barcelonaises étaient les suivantes: Famagouste, Rhodes, Candie, Modon, la Sicile (Syracuse), la Sardaigne (Cagliari) et Majorque ${ }^{39}$. On peut ainsi en déduire que les nouvelles de Damas ont été diffusées dans les principaux centres commerciaux méditerranéens entre décembre 1400 et janvier 1401 hors des circuits de la compagnie Datini et elles ont dû probablement arriver à Barcelone et d'autres villes d'Occident avant les dates indiquées dans les lettres

${ }^{39}$ Coulon 2004, pp. 196-197. 
marchandes de la compagnie Datini. De plus, les lettres n'indiquent pas la date de l'arrivée du navire barcelonais à Candie: avec son arrivée, à partir de Beyrouth le 18 décembre 1400, commença à circuler la nouvelle annonçant que Damas était envahie et incendiée, alors que Tamerlan n'était pas encore arrivé aux alentours de Damas pour l'assiéger avant de l'attaquer: le 6 ğumād al-awwal 803/23 décembre 1400, le sultan Farağ entra à Damas alors que l'armée de Tamerlan était encore dans la plaine de la Bekaa en route vers la ville. Les premiers affrontements entre les deux armées aux alentours de Damas ne commencèrent que le 18 gumāal al-awwal 803/4 janvier $1401^{40}$. Mais il est très probable que durant la durée de voyage de Beyrouth à Candie, et avant leur arrivée dans cette île, les Barcelonais avaient reçu la nouvelle de la prise de Damas en janvier transmise par un autre navire ayant quitté Beyrouth après le 18 décembre 1400. Les Catalans n'étaient pas les seuls à emprunter cette route maritime reliant l'Occident au Levant, et Vénitiens, Génois, Provençaux et Languedociens l'utilisaient également, ce qui accélérait la vitesse de la transmission des lettres et agrandissait le rayon de la diffusion des nouvelles.

Les échanges des lettres concernant la situation en Syrie se sont poursuivis jusqu'en juillet 1401 au sein du réseau Datini installé dans les villes européennes, en vu de suivre le déroulement accéléré des événements et leur impact sur les activités commerciales. Ces lettres ne témoignent pas seulement de la simple transmission de la nouvelle de la prise de Damas par Tamerlan dès les premiers jours de janvier 1401 ; en effet une lettre rédigée par les agents de la compagnie Datini à Alexandrie le 13 février 1401 fait état de la fuite du sultan Farağ et son arrivée au Caire le 27 Janvier $^{41}$; les lettres qui circulaient entre la fin de l'hiver et le printemps 1401, indiquaient que la citadelle de Damas -qui résistait encore après la prise de la ville aux premiers jours de janvier 1401-, était à son tour tombée en février, puis qu'après avoir rançonné les habitants, Tamerlan avait fait incendier la ville en mars; puis qu'il était parti vers Gaza et menaçait l'Égypte en mai. Mais il semblait alors que les nouvelles de la Syrie arrivant en Europe n'étaient ni certaines, ni claires, au moins en janvier et février, ce qui poussa les autorités vénitiennes à prendre des mesures pour réorganiser le commerce avec la Syrie. Le 27 janvier 1401, le Sénat vénitien donna l'ordre au consul de Damas réfugié à Famagouste de retourner à son poste si la situation de la ville s'est rétablie ${ }^{42}$. Et le 22 février

\footnotetext{
${ }^{40}$ Al-Maqrīzī 1997, vol. VI, p. 47.

41 "Sendo il Soldano nele contrado di Domasco, secondo si dice, e vediendo non potere resistere alla gran possanza di Tamberlano, dubitando di non eser rinchiuso e preso dalla giente di Tamberlano, quello ochultamente e con pocha giente fugi di Suria e guinse al Chayro, a dí 27 di giennaio, e da poi giunse quasi tutta la sua giente al Chayro". Ainaud 1965, doc. 1, p. 332.

${ }^{42}$ Archivio di Stato di Venezia, Senato Misti, reg. 45, f. 49 r ${ }^{\circ}$.
} 
1401, ce même Sénat prit des mesures relatives aux marchands de Venise qui avaient quitté la Syrie sans régler leurs comptes ${ }^{43}$. L'embarras et l'inquiétude se manifestèrent dans plusieurs délibérations du Sénat vénitien au cours de l'hiver 1401: on s'interrogea sur les événements de Syrie, attaquée et pillée par Tamerlan et sur ceux d'Égypte, et l'on reprocha aux consuls vénitiens de Damas et d'Alexandrie d'avoir fui l'invasion en se retirant à Famagouste et Candie. Pour le Sénat vénitien, mieux valait rester sur place pour recueillir le plus de renseignements sur les mouvements et les intentions de Tamerlan ${ }^{44}$.

\section{LES RÉPERCUSSIONS DE LA NOUVELLE SUR LES MARCHÉS DES ÉPICES EN EUROPE}

Les épices occupaient la place principale dans le commerce des villes européennes avec le Levant. Par "épices", on entendait au Moyen Âge bien des produits de toute nature et de toute origine, dont certains venaient de l'Orient méditerranéen et d'autres d'Extrême-Orient, apportés par des caravanes terrestres et maritimes. Au Moyen Âge, le terme "épices" englobait les produits condimentaires, pharmaceutiques et tinctoriaux, mais aussi la cire, le sucre, la verrerie et autres produits et articles: il suffisait donc qu'une marchandise provienne d'Orient pour qu'elle soit qualifiée d'épice. Mais au sens étroit du terme, et selon qu'il ait été mentionné dans les livres de comptes des marchands ou défini par les chroniqueurs, ce terme désigne plus restrictivement l'ensemble des innombrables drogues condimentaires, tinctoriales et pharmaceutiques, que fournissait l'Extrême-Orient et les pays de l'Océan Indien, et qui parvenaient aux mains des marchands européens par l'intermédiaire des Arabes, notamment le poivre, le gingembre, le girofle, la cannelle, la noix muscade, l'indigo, etc., le poivre détenant un prestige plus considérable que les autres ${ }^{45}$.

La nouvelle de l'invasion de Damas par Tamerlan produisit une panique dans les villes marchandes européennes par crainte de voir apparaître de très grands désordres sur les marchés des épices. Les marchands européens partirent de la Syrie et rentrèrent dans leurs pays, subissant de lourdes pertes: les marchands vénitiens à Damas et dans les autres villes syriennes perdirent leurs marchandises évaluées à une valeur de 50000 florins $^{46}$. Le consul catalan Pere de Quintana avec sa suite, prit la fuite de Damas vers Beyrouth, face

\footnotetext{
${ }^{43}$ Archivio di Stato di Venezia, Senato Misti, reg. 45, f. 59 r ${ }^{\circ}$.

${ }^{44}$ Thiriet 1978, p. 62.

${ }^{45}$ Moukarzel 2010, p. 406.

${ }^{46}$ Datini, n. 797, Comp. di Simone di Lappacino, 15. 1. 1401.
} 
à l'avance de l'armée de Tamerlan, portant avec lui des marchandises et des biens d'une valeur de quatre mille florins; arrivé dans la plaine de la Bekaa, il fut dévalisé par un émir envoyé par le sultan ${ }^{47}$. Par ailleurs, les complications du commerce international déterminèrent une tendance à la hausse des prix pour de nombreux de produits orientaux, le coton en particulier ${ }^{48}$. Mais à Gênes, la nouvelle fut reçue avec prudence et doute par crainte que les Vénitiens, qui furent les premiers à l'avoir diffusée, aient exagéré ou bien masqué la réalité, profitant de la perturbation des marchés pour faire monter les prix des épices ${ }^{49}$. En effet, les premières nouvelles de la Syrie et du péril menaçant Damas furent diffusés en Europe par les Vénitiens avec l'arrivée d'une lettre envoyée par le consul vénitien de Damas le 11 novembre 1400 qui avait quitté la ville pour se réfugier à Famagouste. Or Damas ne fut prise qu'en janvier et ne fut détruite et incendiée qu'à partir du mercredi $30 \mathrm{rağab}$ 803/1401 jusqu'à $3 \check{s} a$ 'bān 803/1401, du 16 au 19 Mars 1401. Dès l'arrivée à Damas des nouvelles de l'invasion d'Alep (11 rabī' al-awwal 803/28 Octobre 1400) et de Hama (14 rabī'al-awwal 803/2 Novembre 1400) par Tamerlan $^{50}$, puis avant le début du siège de Damas et les premiers affrontements dans ses alentours, les Vénitiens évacuèrent la Syrie en novembre. Ils anticipèrent les événements prévoyant pour Damas les mêmes dévastations que celles subies par les autres villes : voulurent-ils profiter de l'ambigüité de la situation en Syrie pour faire circuler en Europe des nouvelles qui pourraient leur servir pour hausser les prix des épices et des produits orientaux, essayant ainsi de compenser leurs pertes et de ruiner les affaires de leurs concurrents? Cette hypothèse ne peut pas être exclue d'autant qu'à la fin du XIVe siècle, la concurrence économique fut très forte entre Venise et les villes commerçantes méditerranéennes, Gênes en tête.

\footnotetext{
${ }^{47}$ Ainaud 1965, doc. 2, p. 333.

${ }^{48}$ Les prix du coton devinrent très élevés à Venise. Datini, n. 797, Comp. di Simone di Lappacino, 15.1 1401. Dès la seconde moitié du XIVe siècle, le commerce du coton en Syrie tomba progressivement sous le contrôle des marchands vénitiens. Le coton syrien offrait de loin la meilleure qualité, surpassant celui d'Égypte. Les navires de Venise chargeaient du coton dans quatre ports, Acre, Beyrouth, Tripoli et Laodicée, alors que les autres villes maritimes européennes le chargeaient presque seulement à Beyrouth. En 1400, les Vénitiens envoyèrent dix coques pour charger le coton syrien durant la muda de mars, et six coques durant la muda de septembre, alors qu'en 1401 le service du transport du coton syrien fut suspendu à cause de l'occupation de Damas par Tamerlan. Pour assurer le ravitaillement de leurs marchés, deux coques vénitiennes chargeaient le coton à Alexandrie en mars 1401. Le coton se trouvait en faibles quantités sur les marchés de Venise durant cette année. Nam 2007, pp. 114-115, 436 , 441. Pour Gênes et Barcelone, les cotons syriens et égyptiens étaient importés régulièrement au tournant de l'année 1400 mais leur volume n'était pas impressionnant. Les deux villes s'approvisionnaient en Sicile, Malte et le sud de l'Italie. ibidem, p. 287 ; Coulon 2004, pp. 466-467.

${ }^{49}$ Datini, n. 881, lett. Genova-Barcellona, Comp. Datini, 20. 2. 1401, c. 2, citée par Melis 1962, p. 30 ; Datini, n. 1071, lett. Genova-Maiorca, Comp. Datini, 20. 2. 1401, ibidem, p. 31.

${ }^{50}$ Ibn Tağrī Birdī 1992, vol. XII, pp. 179-181.
} 
L'installation de puissantes compagnies commerciales à Gênes, la concurrente principale de Venise, attirées à cette époque par une large participation internationale au grand commerce des épices, jusqu'alors réservé en très grande partie aux Vénitiens, contribua à l'amplification de la concurrence commerciale qui en résulta. Ainsi, la nouvelle voit élargir son rayon d'action et accroître l'intensité de son action économique. La répercussion économique de l'annonce de la destruction de Damas qui fut le principal marché des épices au sud de la Syrie, s'intensifia quand vint, d'Alexandrie et de Rhodes, la confirmation de l'événement par une lettre des agents de la compagnie Tecchini. De plus, les marchands rentrant du Levant annoncèrent que la situation en Syrie empirait jour après jour et que l'armée de Tamerlan composée de 600.000 hommes, après avoir détruit et incendié Damas, avait pris la direction de Gaza et d'al-Ġawr avec le but d'attaquer le Caire ${ }^{51}$. À la suite d'une telle nouvelle, le commerce des épices subit de graves troubles: les navires européens partant des ports levantins rentrèrent avec des quantités d'épices inférieures à celles chargées durant les années précédentes, ou à vide sans épices, et les marchands ne voulurent plus vendre leurs stocks d'épices et de coton en attendant l'éclaircissement de la situation ${ }^{52}$. À Venise, au principal marché des épices, les quantités vendues furent très minimes et varièrent chaque jour: d'après une lettre envoyée de Gênes à Florence le 25 février, une certaine quantité de poivre y fut vendue à 28 livres, du girofle à 18 sous, du coton à 25 livres $^{53}$. Les nouvelles qui arrivèrent aux centres commerciaux provoquèrent une montée considérable des prix entraînèrent tout de suite la spéculation.

Le fonctionnement des marchés était fondé sur un échange rythmé entre produits en provenance de l'Orient et produits européens; il s'accomplissait chaque année par l'arrivée au Levant des vaisseaux apportant et déchargeant des marchandises occidentales et qui revenaient ensuite de l'Orient chargés d'épices et des différents produits orientaux. La relative stabilité des prix sur les marchés européens, en particulier de Venise, Gênes et Barcelone, des principales épices, poivre, gingembre, cannelle et clous de girofle, s'explique sans doute par la régularité des approvisionnements en épices entre

\footnotetext{
51 “Abiam lettera noi da Rodi, di dì primo di maggio, e cierto dicie aveano che'l Tabarlencho era partito da Damasco e tutto l'avea afochato e messo a terra el castello e tutto; e fatte gran crudeltà di instizie ed era tra'l Ghaor e Ghazera, ch'andava al Chairo con più di 600 mila di persone". Datini, n. 1072, lett. Iviza-Maiorca, Tucci di Gennaio, 29.4(1.5). 1401, c. 2t; Datini, n. 906, lett. Perpignano-Barcellona, Pietro Tecchini, 10. 7. 1401, c. 1t, citées par Melis 1962, p. 32 .

${ }^{52}$ Datini, n. 777, lett. Gaeta-Genova, Comp. d'Agnolo di ser Pino e Giuliano di Giovanni Portinari, 22.2.1401, ibidem, p. 32.

53 "Ed èsi venduto alquanto pepe a lb.28, ma poca cosa; garofani, s.18; i cotoni tenghono a lb.25. Varà tutte spezie de meglo ogni dì”. Datini, n. 662, lett. Genova-Firenze, Comp. Datini, 25.2. 1401, ibidem, p. 31. (La livre $=10$ ducats $=20$ sous).
} 
les deux rives de la Méditerranée ${ }^{54}$. Toute perturbation de ce cycle réelle ou fabriquée, pouvait avoir des répercussions sur le commerce et de graves conséquences financières et politiques, qui permettaient aux spéculateurs, jouant sur les craintes, d'augmenter les prix et de fausser les conditions du marché des produits les plus appréciés. La destruction de Damas affecta réellement pour un temps la stabilité des échanges: par exemple, le cours du poivre à Damas entre 1392 et 1399 variait entre 50 et 90 dīnār le qintār $r$, mais en 1400-1401 le prix d'un qintār de poivre atteignit 141 puis 170 dīnār, il ne baissa à 69,3 puis 43,3 dīnār qu'à partir de $1402^{55}$. Les navires européens provenant de Beyrouth et d'Alexandrie portant des épices jouaient un rôle primordial dans le maintien de la stabilité de l'économie des principales villes marchandes. Les prix des marchés des épices variaient selon la date d'arrivée des navires et les quantités d'épices et de produits orientaux chargées à Beyrouth et à Alexandrie. À Venise, selon des remarques faites par le marchand Michiel Contarini sur le déroulement des affaires, si les galères tardaient à rentrer ou si elles rentraient sans grandes quantités d'épices, les prix ne cessaient pas de monter par rapport au prix normal du marchés6.

À Venise, le voyage annuel du convoi des galères (la muda) jusqu'au port de Beyrouth, le port le plus proche de Damas, pour charger les épices et les produits de luxe, fut annulé en $803 / 1401^{57}$. Cette interruption causa une grave perturbation dans les affaires parce qu'aux arrivées de galères marchandes en provenance de Beyrouth et d'Alexandrie étaient liées d'importantes opérations commerciales à Venise, notamment la foire de Noël, c'est-à-dire une période d'intense activité des échanges qui s'étendait de la mi-décembre jusqu'à la mi-février ${ }^{58}$. Il est vrai que si les convois de galères de Beyrouth, organisés par le Sénat vénitien, furent interrompus en 803/1401, mais les navires privés des marchands vénitiens et ceux des marchands européens continuèrent

${ }^{54}$ Heers 1955, pp. 202-205 ; Coulon 2004, pp. 440-452; Wake 1979, pp. 364-371.

${ }^{55}$ Ashtor 1983, pp. 546-547.

${ }^{56}$ Archivio di Stato di Venezia, Miscellanea Gregolin, busta 8. On peut citer un autre exemple de l'instabilité du système des galères et ses conséquences sur les marchés de Venise des années 1448 et 1449: Le Sénat vénitien interrompit le voyage des galères vers Beyrouth et Alexandrie en 1448, et l'année suivante, les galères rentrèrent vides; il résulta un manque à gagner d'un million de dīnār de marchandises dans ces deux ans. Apellániz 2009, p. 162.

${ }^{57}$ Archivio di Stato di Venezia, Senato Misti, reg. 45.

${ }^{58} \mathrm{Il}$ n'y a pas une période fixe pour les ventes et les achats à Venise: les marchés sont ouverts et fréquentés par des marchands étrangers tous les jours de l'année. Mais il y a à Venise trois foires importantes: la foire de l'Ascension qui dure 15 jours; la foire de Noël qui coïncide avec l'arrivée des galères du Levant (Beyrouth et Alexandrie) chargées d'épices; la foire de juillet mentionnée par Priuli la fiera consuetta deli Todeschi di San Jacomo durant laquelle les marchands allemands fréquentent les marchés de Venise pour l'achat des épices. (La fête de San Jacomo est le 25 juillet). Priuli 1921, pp. 382, 431. Pour des informations sur les foires et leur déroulement à Venise, voir: Luzzatto 1954, pp. 201-209. 
à fréquenter les ports du littoral syrien, et en particulier le port de Beyrouth. En 803/1401, d'après les registres des archives Datini, le nombre de navires au port de Beyrouth fut réparti comme suit ${ }^{59}$ :

\begin{tabular}{|l|c|}
\hline \multicolumn{1}{|c|}{ VILLES MARCHANDES } & NOMBRE DE NAVIRES \\
\hline Biscaye & 1 \\
\hline Catalogne & 14 \\
\hline Provence & 3 \\
\hline Gênes & 16 \\
\hline Venise & 22 \\
\hline Perpignan & 2 \\
\hline
\end{tabular}

Ce tableau montre que les épices et les produits provenant de la Syrie, n'avaient pas disparu complètement des marchés européens en 803/1401, et particulièrement des marchés vénitiens, génois, et catalans, mais que leurs quantités avaient diminué, causant une hausse des prix et un dérèglement des affaires. Cette situation poussa les marchands européens à diriger une grande partie de leurs affaires vers les marchés égyptiens où ils pouvaient s'approvisionner en épices. Les agents de la compagnie Datini à Alexandrie en 803/1401 donnèrent, par une lettre écrite le 16 février, des informations sur les quantités d'épices chargées à bord des navires européens, et sur les fluctuations des prix de marchandises sur les marchés d'Alexandrie. Les achats des marchands génois à Alexandrie en 803/1401 furent comme suit ${ }^{60}$ :

\begin{tabular}{|l|c|c|}
\hline \multicolumn{1}{|c|}{ MARCHANDISES } & QUANTITÉS & VALEUR EN KILOGRAMMES \\
\hline poivre & 1.000 sporte & $210.000 \mathrm{~kg}$ \\
\hline gingembre beledi et colombini $^{61}$ & 1.400 cantari & $63.000 \mathrm{~kg}$ \\
\hline gingembre micchino $^{62}$ & 200 cantari & $9.000 \mathrm{~kg}$ \\
\hline laque & 1.000 cantari & $45.000 \mathrm{~kg}$ \\
\hline cannelle & 200 fardi & $28.000 \mathrm{~kg}$ \\
\hline
\end{tabular}

${ }^{59}$ Melis 1970, p. 373.

${ }^{60}$ Ainaud 1965, doc. 1, p. 332.

${ }^{61}$ Le mot beledi dérive de l'arabe balad (pays), c'est le nom que les musulmans installés dans l'Inde donnent au gingembre indigène. Le mot colombino ainsi nommé parce qu'il croît dans une ville de l'Inde Columbum sur la côte de Malabar. Heyd 1959, vol. II, pp. 619-29.

${ }^{62}$ La sorte de gingembre qui provient des environs de la Mecque est appelée micchino. Ce n'est pas un produit du territoire mecquin, mais un article du marché de la Mecque. Le gingembre micchino est toujours considéré comme gingembre de la Mecque, mais cette plante n'existe pas en Arabie. Il s'agit non de provenance mais du grand marché de distribution au Levant qui se trouve à la Mecque. Heyd 1959, vol.II, pp. 619-29; Magalhães-Godinho 1969, p. 585. 
En outre, les marchands génois achetèrent d'autres produits. La somme des investissements des Génois à Alexandrie en 803/1401 fut évaluée à 80.000 besants $(d \bar{\imath} n \bar{a} r)$, monnaies d'or et marchandises, payant en espèces et en troc. Trois navires génois partirent d'Alexandrie en Mars: deux vers les Flandres et un vers la Provence; toutes les cargaisons de poivre furent envoyées en Flandres, tandis que les cargaisons de gingembre, de cannelle et de laque prirent la direction de l'Espagne. Ils envoyèrent le reste des marchandises vers Gênes et la Provence ${ }^{63}$.

Les investissements des marchands vénitiens à Alexandrie en 803/1401 atteignirent 50.000 besants (dīnār), et il resta à investir $22.000^{64}$. Deux navires vénitiens arrivèrent à Candie portant chacun 500 pondi (1 pon$d o=180 \mathrm{~kg}$ ) d'épices chargées à Alexandrie ${ }^{65}$.

Une coque catalane partit d'Alexandrie en mars vers Majorque et Barcelone portant une cargaison de marchandises mais qui n'était pas assez grande, et deux autres navires arrivèrent à Alexandrie portant de l'huile et des draps de laine.

Les marchands musulmans à Alexandrie n'arrivèrent pas à vendre toutes les sortes d'épices qu'ils possédaient. Il resta au marché d'Alexandrie 800 à 1.000 sporte de poivre, 200 sporte de gingembre colombino et beledi, une quantité de gingembre micchino, 200 à 400 sporte de gingembre mais elles sont trop mouillées. Il ne resta que de petites quantités de gingembre micchino et d'épices légères, et de bonnes quantités de cannelle, de laque, d'indigo, de bois du brésil, de bois de santal, d'encens. Au Caire, il ne resta que peu de marchandises. En 1401, la caravane d'épices arrivée au Sinaï porta une charge de 10.000 pondi d'épices, mais elle ne continua pas son chemin vers le Caire à cause des nouvelles des victoires de Tamerlan et de ses préparatifs pour attaquer l'Égypte. En outre, les transactions commerciales à Alexandrie connaissant des troubles, les prix de tous les produits européens baissèrent, le ducat d'or garda sa valeur (22 carats) mais le dīnār d'or perdit de sa valeur : il valut 34 dirhams, mais à cause de la crise économique résultant de l'invasion de Tamerlan, sa valeur devint 37 dirhams $^{66}$.

\footnotetext{
${ }^{63}$ Ainaud 1965, doc. 1, p. 332.

${ }^{64}$ Les achats des Vénitiens à Alexandrie augmentèrent progressivement et passèrent de 72000 dīnār $r$ en février 1401 à 303,097 dīnāa $r$ en septembre de la même année, dépassant de loin les achats effectués à Beyrouth et Alexandrie dans les années allant de 1382 à 1408. Ashtor 1975, pp. 580-582. Cette hausse s'explique probablement par l'essai des marchands vénitiens de compenser leurs pertes dues aux achats très réduits des épices et d'autres produits orientaux aux ports syriens en 1400-1401 à la suite de l'invasion de Tamerlan. Ils voulaient assurer le maintien de l'équilibre du fonctionnement du marché de Venise.

${ }^{65}$ Le convoi partant de Venise pour la muda d'Alexandrie en 1401 comprenait trois galères. Stöckly 1995, p. 136.

${ }^{66}$ Ainaud 1965, doc. 1, pp. 332-333.
} 
Pour les monnaies d'argent, on employait en Syrie beaucoup plus qu'en Égypte des dirhams d'argent comme moyen de paiement. Le développement du monnayage d'argent en Syrie et en Égypte était différent jusqu'à la fin du règne des Mamelouks. De plus, il y avait dans les diverses provinces de la Syrie des dirhams se distinguant par leur poids, leur aloi et leur taux de change. La pénurie d'argent au début du règne des Mamelouks circassiens en 784/1382 contraignit le gouvernement à frapper des monnaies dépréciées dans la dernière décennie du XIV siècle $^{67}$. Al-Qalqašandī mentionne que durant cette période la frappe de monnaie d'argent en Égypte avait pratiquement cessé, mais que les autorités locales en Syrie avaient continué à frapper les dirhams, bien qu'ils aient été de mauvaise qualité68. Selon al-Maqrīzīi, la valeur du dīnār en dirham variait comme suit: 30 en 801/1399 et 802/1400, 40 en 803/1401, 50 en 804/1402 et 65 en 805/1403 ${ }^{69}$. En 1400, les autorités mameloukes s'engagèrent en vain dans la restauration du rôle du dirham dans leur système monétaire ${ }^{70}$. L'invasion et le pillage systématique de Tamerlan accélérèrent la dépréciation des monnaies et firent diminuer les stocks d'argent en Syrie. Tamerlan collecta tous les stocks d'argent qui se trouvaient à Damas et installa un atelier monétaire de campagne qui avait plusieurs fonctions: raffiner l'argent pour alléger le poids du butin et de le reconvertir dans une forme plus appropriée, gagner des revenus pour la trésorerie de l'envahisseur et servir d'outil de propagande pour des nouvelles conquêtes en émettant des monnaies portant son nom. Le montant d'argent emporté devait être énorme, comprenant la quasi-totalité du stock de métal précieux d'au moins trois grandes villes syriennes, Alep, Hama et Damas, affectant la circulation et le système monétaire en Syrie et en Égypte ${ }^{71}$. Ce n'est qu'en 814/1412 que l'émir Nawrūz frappa à Damas des demi-dirhams qui étaient d'argent pur, puis en 817/1415, la frappe de dirhams de bon aloi fut reprise en Égypte ${ }^{72}$.

Bien que l'occupation de Damas par Tamerlan n'ait duré que 80 jours, elle laissa de graves séquelles sur le fonctionnement des marchés.

\footnotetext{
${ }^{67}$ Ashtor 1978, p. 276. Les stocks d'argent dans le sultanat mamelouk avaient diminué à partir des années 1380 par suite de son exportation en Europe, les guerres civiles, la thésaurisation, et l'envoi continu de l'argent et d'autres métaux en direction de l'Inde. Bacharach 1994, pp. $98-100$.

${ }^{68}$ Al-Qalqašandī 1918-1922, vol. III, p. 467. Ces dirhams devaient être frappés en quantités énormes. Shultz 1995, pp. 159-162.

${ }^{69}$ Al-Maqrīzī 1997, vol. V, p. 452; vol. VI, p. 37, 60, 70, 90. Pour des informations sur la situation économique en Égypte au début du XV siècle, voir: Apellániz 2009, pp. 67-68.

${ }^{70}$ Bacharach 1971, p. 280.

${ }^{71}$ Heidemann 1999, pp. 179-208. Tamerlan prit également de Damas des quantités considérables de dīnār et dirhams, environ dix millions dīnār. Al-Maqrīzī 1997, vol. VI, pp. 51-52; Fischel 1954, p. 221.

${ }^{72}$ Ashtor 1983, p. 276; Balog 1961, p. 142.
} 
La Syrie située sur les axes de différentes routes commerciales: route de la Perse et de l'Asie mineure, route du Golfe à travers l'Irak, route de la péninsule Arabique, apparut comme une contrée largement intégrée à un vaste ensemble régional. Profitant des changements politiques, militaires et commerciaux qui ont marqué le XIVe siècle, la Syrie réussit à attirer une grande partie des courants commerciaux qui transitaient autrefois par la mer Noire ou à travers l'Asie centrale. Elle n'était pas seulement intéressante pour les Européens par sa position d'intermédiaire sur les routes du commerce international, mais certaines productions locales, comme le coton et les cendres ${ }^{73}$, étaient achetées en quantités importantes. Ainsi, les principales villes syriennes formaient-elles la plaque tournante centrale du commerce entre l'Occident et l'Orient. L'axe Damas-Beyrouth devenait alors un centre important du commerce international de l'empire mamelouk : d'une part, les caravanes apportaient à Damas les épices, les soieries, le coton, qui étaient ensuite expédiés à Beyrouth en tant que marchandises de transit ou exportation de l'Est vers l'Ouest, d'autre part les navires européens déchargeaient à Beyrouth des produits divers, surtout les métaux et les draps, en tant que marchandises de transit ou d'importation de $l^{\prime} O u e s t$ vers $l^{\prime} E s t^{74}$. Au cours des deux dernières décennies du XIV ${ }^{\mathrm{e}}$ siècle, le nombre de navires européens appareillant pour Beyrouth croît continuellement, et ce port finit par dépasser Alexandrie pour le volume du trafic avec les principales villes marchandes européennes durant les années 1390. Les registres du Sénat vénitien entre 1391 et 1400 mentionnent l'arrivée de 46 galères à Beyrouth contre 29 galères à Alexandrie ${ }^{75}$. De même, une lettre écrite de Damas, le 2 août 1395, par Beltramo Mignanelli, un agent de la firme Datini, indique clairement que l'année 1395 est un tournant très important pour les marchands européens impliqués dans le commerce des épices. D'après Mignanelli, en 1395, depuis 25 ans il n'a pas vu autant de marchands européens, particulièrement des marchands catalans, génois et vénitiens, à Damas et à Beyrouth. Ils ont importé de grandes quantités d'épices malgré les prix très élevés des produits ${ }^{76}$. Entre 1397 et 1399 , quatorze navires catalans arrivaient à Beyrouth contre neuf vers Alexandrie, sans compter ceux qui avaient les deux villes pour destination ${ }^{77}$. L'essor économique fut interrompu pour quelques mois avec la destruction de Damas, objectif réel des voyages pour Beyrouth.

${ }^{73}$ Il s'agit de trois espèces de plantes réduites en cendres qui donnent un produit utilisé dans la fabrication du savon et du verre. Les cendres de Syrie étaient de meilleures qualités que celles provenant d'Alexandrie; elles se vendaient sous forme de grosses pierres, et non en poudre, chargées dans des sacs de canevas. Pegolotti 1936, pp. 379-380.

${ }^{74}$ Moukarzel 2010, p. 35.

${ }^{75}$ Ibidem, p. 320.

${ }^{76}$ Melis 1972, p. 184.

${ }^{77}$ Coulon 2004, p. 190. 
Cet événement constitua le début du recul progressif du trafic européen vers Beyrouth, le port de Damas, en faveur du développement des voyages vers Alexandrie. Le raid de Tamerlan en Syrie modifia les caractères du trafic avec le Levant : les galères vénitiennes importaient toujours les épices et certaines étoffes, mais elles emportaient à présent des céréales, du sucre, du bois, des draps, des soieries et des cotonnades. Les navires génois firent augmenter les expéditions de l'huile d'olive et des grains. Ce changement fut le résultat $\mathrm{du}$ fort déclin de la production syrienne après l'invasion de Tamerlan ${ }^{78}$. De même, les exportations catalanes de miel, de fruits secs, d'huile d'olive occupèrent une part de plus en plus importante dans les cargaisons des navires fréquentant Beyrouth, mais le safran se repliait vers d'autres destinations tandis que l'activité florissante de l'exportation de corail de Barcelone vers Beyrouth fut interrompue, et à partir de 1401, Alexandrie devint le terme de nombreuses commandes catalanes investies dans le corail ${ }^{79}$.

Les informations fournies par les lettres des agents de la compagnie Datini installés à Alexandrie prouvent que l'invasion de la Syrie par l'armée de Tamerlan n'eut pas des conséquences catastrophiques directes pour l'ensemble des principales villes marchandes européennes qui avaient des activités commerciales dans le sultanat mamelouk. Le commerce méditerranéen subit une perturbation de quelques mois, qui s'explique à la fois par l'altération du caractère commercial saisonnier des affaires et par l'action contingente des nouvelles relatives à la conjoncture politique internationale. Cette difficulté une fois franchie, la situation ne tarda pas à reprendre son cycle normal. Il est certain que la destruction de Damas a bouleversé les marchés des épices, la valeur des monnaies locales et les prix dans le sultanat mamelouk, et d'un autre côté fit monter les prix sur les marchés européens, suscitant les spéculations, mais les principaux ports levantins au tournant du $\mathrm{XV}^{\mathrm{e}}$ siècle, Beyrouth et Alexandrie, continuèrent d'une part, à assurer une partie des besoins des Européens en produits orientaux, et d'autre part permirent aux marchands orientaux de maintenir le déroulement de leurs affaires malgré les changements économiques, politiques et sociaux qui eurent lieu à l'intérieur du sultanat mamelouk. Les échanges commerciaux opérés entre

\footnotetext{
${ }^{78}$ Thiriet 1978, p. 67; Ashtor 1983, p. 482. Après l'invasion de Tamerlan, la production syrienne déclina fort: Damas, Alep et Hama, les principales villes marchandes syriennes, furent détruites et perdirent la moitié de leur population; la production agricole à Damas fut ravagée durant plusieurs jours par une grande vague de sauterelles; les bédouins pillèrent les villes et les campagnes syriennes. Al-Maqrīzī 1997, vol. VI, pp. 58, 63, 65. En outre, beaucoup d'artisans damascènes étaient déportés à Samarcande par Tamerlan. En 1449, le pèlerin allemand Stephan von Gumpenberg passa à Damas et apprit que les soieries arrivaient de Venise, parce que Tamerlan avait emmené tous les maîtres ouvriers. Heyd 1959, vol. II, p. 469.

${ }^{79}$ Coulon 1996-1997, pp. 144-148; Coulon 2004, p. 424.
} 
les deux extrémités de la Méditerranée montrent que l'Europe et l'Orient étaient tributaires l'un de l'autre.

\section{CONCLUSION}

La transmission des nouvelles constituait un enjeu pour les marchands dans la gestion des affaires et fut un élément fondamental dans la vie d'une société commerciale à la fin du Moyen Âge. L'échange de lettres permit aux marchands d'être au courant des conditions de vente et d'achat dans les places étrangères, et d'éviter les risques qui menacèrent le bon déroulement de leurs activités commerciales. Le partage de nouvelles venant de tout le bassin méditerranéen était une fonction essentielle des associations commerciales entretenues dans le but d'avoir des avantages mutuels et d'assurer la prospérité de leurs membres. Les correspondances ne comprirent pas seulement des informations, mais aussi des réponses et offrirent des remarques sur les décisions à prendre. La nouvelle que constitua l'invasion de Tamerlan en est un bon exemple de la fonction informative des lettres transmettant une information d'importance dans des milieux marchands, nouvelle de nature à modifier plus ou moins profondément et durablement les codes et les activités commerciales et de ce fait à avoir une large répercussion dans les milieux où elles arrivaient. La mention épistolaire d'un événement de plus ou moins grande portée prouvait toujours que l'auteur de la lettre avait estimé qu'il pourrait avoir une certaine importance et incidence sur les affaires en cours ou à venir avec son correspondant. Cependant, la transmission d'une nouvelle était seulement l'une des fonctions de ces lettres qui étaient en effet, avant tout, un dialogue suivi entre l'expéditeur et le destinataire, concernant leurs investissements particuliers et les conditions du déroulement de leurs affaires.

La nouvelle de l'invasion de la Syrie en 802/1400-803/1401 causa une certaine panique dans les principaux centres commerciaux européens, mais ses séquelles furent limitées puisque les marchés d'Alexandrie, de Beyrouth et du littoral syrien restèrent ouverts aux marchands, et en 804/1402 les affaires commerciales reprirent leur élan normal ${ }^{80}$. En 803/1401, les marchands européens réduisirent prudemment leurs activités dans les ports syriens, bien que les nouvelles, attestant que Tamerlan était parti de Damas, aient déjà été reçues en Europe au mois de mai ${ }^{81}$. Après Damas, Tamerlan se dirigea au printemps 1401 vers l'Égypte, avant de repartir ensuite vers l'Orient. La circulation de la

\footnotetext{
${ }^{80}$ Archivio di Stato di Venezia, Senato Misti, reg. 46, f. 31rº.

${ }^{81}$ Tamerlan partit de Damas le samedi 3 ša'bān 803 /19 Mars 1401. Ibn Tağrī Birdī 1992 , vol. XII, p. 195; Al-Maqrīzī 1997, vol. VI, p. 58.
} 
nouvelle annonçant l'intention de Tamerlan d'envahir l'Égypte causa la perturbation des marchés, mais cette situation ne fut que de courte durée.

Quant à Damas, l'économie reprend péniblement suite à la déportation d'une partie des maîtres-artisans par Tamerlan à Samarcande ${ }^{82}$. Alors, l'activité marchande se déplaçait aux faubourgs de la ville. D'après alMaqrīzī, le 26 rağab 804/31 Mars 1402, la prière du vendredi fut élevée dans la mosquée des Omeyyades détruite pendant l'incendie causé par Tamerlan. La mosquée et l'ensemble de la ville furent transformés en ruine. Les Damascènes se déplacèrent en dehors de la ville pour reprendre le cours de leur vie et construisirent des maisons par les pierres des monuments détruits; les traces de l'incendie de Damas disparaissaient progressivement ${ }^{83}$. La restauration de la mosquée et de ses alentours par les sultans dès 804/1402 visait à amorcer un retour rapide de la population et redonner vie au commerce damascène ${ }^{84}$. Damas renaquit de ses cendres et reprit sa place alors que le sultanat mamelouk au début du $X V^{e}$ siècle était plongé dans une crise économique sans précédent aggravée par une instabilité politique ${ }^{85}$.

L'invasion de la Syrie par Tamerlan avait laissé des effets catastrophiques sur les principales villes syriennes: après 80 jours d'occupation, les troupes de Tamerlan quittèrent Damas non sans l'avoir pillée et incendiée. Mais après le retrait de l'armée de Tamerlan, les activités intenses des marchands européens en Syrie et en Égypte continuèrent comme auparavant ${ }^{86}$. Tandis que les Mamelouks avaient essayé de reconstruire leur gouvernement ébranlé, les villes marchandes européennes jouissaient d'une suprématie incontestée sur mer, et leur puissance navale assura le succès de leurs activités commerciales.

\section{BIBLIOGRAPHIE}

Al-Qalqašandī (1918-1922), Subh al-a 'šā fì sināa at al-inšā, vol. III, Le Caire, Ibrahim Muhammad 'Āšūr.

Ainaud, Joan (1965), Quatre documents sobre el comerç català amb Síria $i$ Alexandria (1401-1410), dans Homenaje a Jaime Vicens Vives, Barcelone, Universidad de Barcelona - Facultad de Filosofía y Letras, vol. I, pp. 327-335.

\footnotetext{
${ }^{82}$ Ibn `Arabšāh 1986, pp.193-194; Fischel 1954, p. 225.

${ }^{83}$ Al-Maqrīzī 1997, vol. VI, p. 75.

${ }^{84}$ Vigouroux 2012, pp. 127-143.

${ }^{85}$ Apellániz 2009, p. 68.

${ }^{86}$ E. Ashtor 1983, pp. 125-126.
} 
Al-Maqrīzī (1997), Al-sulūk li-ma'rifat duwal al-mulūk, vol. V et VI, Beyrouth, Muhammad 'Abd al-Qādir 'Atā, Dār al-kutub al-'ilmiyya.

Apellániz, Francisco (2009), Pouvoir et finance en Méditerranée pré-moderne: le deuxième État mamelouk et le commerce des épices (13821517), Barcelone, Consejo Superior de Investigaciones Científicas.

Ashtor, Eliyahu (1983), Levant trade in the later Middle Ages, Princeton, Princeton University Press.

Ashtor, Eliyahu (1978), Études sur le système monétaire des Mamlouks circassiens, dans The Medieval Near East: Social and economic history, Londres, Variorum reprints, pp. 264-287.

Ashtor, Eliyahu (1975), The volume of Levantine trade in the later Middle Ages (1370-1498), "Journal of European Economic History" 4, pp. 573-612.

Bacharach, Jere L. 1994, The ducat in fourteenth century Egypt, dans Itinéraires d'Orient. Hommages à Claude Cahen, vol. VI, pp. 95-101.

Bacharach, Jere L. (1971), Circassian monetary policy: Silver, "Numismatic Chronicle" 11, pp. 267-281.

Balog, Paul (1961), History of the dirhem in Egypt from the Fatimid conquest until the collapse of the Mamluk empire 358H-922H/968AD-1517AD, "Revue Numismatique" 3, pp. 109-146.

Bauden, Frédéric (2010), D'Alexandrie à Damas et retour. La poste privée à l'époque mamelouke à la lumière d'une commission accomplie pour le compte d'un vénitien (821 A.H./1418 È.C.), dans Egypt and Syria in the Fatimid, Ayyubid and Mamluk eras, VI, Proceedings of the $14^{\text {th }}$ and $15^{\text {th }}$ International Colloquium organized at the Katholieke Universiteit Leuven in May 2005 and May 2006. Leuven, Uitgeverij Peeters, pp. 157-189.

Casola, Pietro (1907), Canon Pietro Casola's pilgrimage to Jerusalem in the year 1494, Manchester, University Press.

Christ, Georg (2011), Quelques observations sur la navigation vénitienne à Alexandrie à la fin du Moyen Âge, dans Venise et la Méditerranée, Venise, Institut National du Patrimoine, pp. 55-72.

Christ, Georg (2010), Passagers clandestins? Rôle moteur des galères vénitiennes et concurrence des navires ronds à Alexandrie au début du $X V^{e}$ siècle, dans Espaces et réseaux en Méditerranée $V I^{e}-X V I^{e}$ siècle: Vol. 2, La formation des réseaux, Paris, Éditions Bouchène, pp. 275290.

Congdon, Eleanor A. (1997), Datini and Venice: News from the Mediterranean trade network, dans Across the mediterranean frontiers. Trade, politics and religion, 650-1450, Turnhout, Brepols, pp.157-171. 
Coulon, Damien (2004), Barcelone et le grand commerce d'Orient au Moyen $\hat{A} g e$. Un siècle de relations avec l'Égypte et la Syrie-Palestine (ca.1330-ca.1430), Madrid, Casa de Velázquez - Barcelone, Institut Europeu de la Mediterrània.

Coulon, Damien (1996-1997), Un élément clef de la puissance commerciale catalane: Le trafic du corail avec l'Égypte et la Syrie (fin du XIV début XV siècles, "Al-Masāq" 9, pp. 99-149.

Doumerc, Bernard (1994), Par Dieu écrivez plus souvent! La lettre d'affaires à Venise à la fin du Moyen Âge, dans La circulation des nouvelles au Moyen Âge. XXIV congrès de la SHMES (Avignon, juin 1993), Paris, Publications de la Sorbonne, pp. 99-109.

Doumerc, Bernard (1991), Le galere da mercato, dans Storia di Venezia. Temi. Il Mare. A cura di Alberto Tenenti e Ugo Tucci, Rome, Istituto della Enciclopedia Italiana, pp. 357-395.

Favier, Jean (1987), De l'or et des épices. Naissance de l'homme d'affaires au Moyen Âge, Paris, Fayard.

Fischel,Walter J. (1954), A new Latin source on Tamerlane's conquest of Damascus (1400-1401) (B. De Mignanelli's "Vita Tamerlani” 1416), "Oriens" 9, pp. 201-232.

Goiten, Shelomo Dov (1999), A Mediterranean society. The Jewish communities of the World as portrayed in the documents of the Cairo Geniza, vol. 1, Californie, Economic Foundations, University of California Press.

Hayez, Jérôme (2004), Avviso, informazione, novella, nuova: la notion de l'information dans les correspondances marchandes toscanes vers 1400, dans Information et société en Occident à la fin du Moyen Âge, Actes du colloque international tenu à l'université du Québec à Montréal et à l'université d'Ottawa (9-11 mai 2002), Paris, Publications de la Sorbonne, pp. 113-134.

Hayez, Jérôme (1994), La gestion d'une relation épistolaire dans les milieux d'affaires toscans à la fin du Moyen Âge, dans La circulation des nouvelles au Moyen Âge. XXIV congrès de la SHMES (Avignon, juin 1993), Paris, Publications de la Sorbonne, pp. 63-84.

Heers, Jacques (1955), Il commercio nel Mediterraneo alla fine del sec. XIV e nei primi anni del $X V$, “Archivio Storico Italiano" 113, pp. 165-209.

Heidemann, Stefan (1999), Tìmūr's campmint during the siege of Damascus in 803/1401, dans Matériaux pour l'histoire économique du monde iranien. Studia Iranica, cahier 21, Paris, L'Association pour l'avancement des études iraniennes, pp. 179-208.

Heyd, Wilhelm (1959), Histoire du commerce du Levant au Moyen Âge, vol. II, Amsterdam, Adolf. M. Hakkert. 
Hocquet, Jean Claude (1991), L'armamento privato, dans Storia di Venezia. Temi. Il Mare, Rome, Istituto della Enciclopedia Italiana, pp. 397-434.

Hocquet, Jean Claude (1979), Le sel et la fortune de Venise, vol. 2. Voiliers et commerce en Méditerranée 1200-1650, Lille, Presses Universitaires.

Ibn 'Arabšāh, (1986), 'A ğă'ib al-maqdūr fī akhbār Tìmūr, Muhammad Fa'iz al-Himsī, Beyrouth, Mu'assasat al-Risāla.

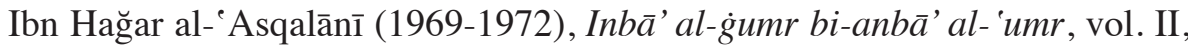
Le Caire, Hasan Habšĩ.

Ibn Tağnī Birdī (1992), Al-nuğūm al-zāhira fī mulūk Misr wa-l-Qāhira, vol. 12, Muhammad Husayn Šams al-Dīn, Beyrouth, Dār al-kutub al'ilmiyya.

Ibn Tag̉ñ̄ Birdī (1986), Al-Manhal al- Șāfî wa-l-mustawfa ba'da-l-wāfĭ, vol. 4, Le Caire, Muhammad Muhammad Amīn.

Judde de Larivière, Claire (2008), Naviguer, commercer, gouverner. Économie maritime et pouvoirs à Venise (XV-XVI siècles), Leiden, Brill.

Lane, Frederic (1963), The economic meaning of the invention of the compass, "American Historical Review", 78/3, pp. 605-617.

Luzzatto, Gino (1954), Navigazione di linea e navigazione libera nelle grande città marinere del Medioevo, dans Studi di storia economica veneziana, Padoue, CEDAM, pp. 53-57.

Luzzatto, Gino (1954) Vi furono fiere a Venezia?, dans Studi di storia economica veneziana, Padoue, CEDAM, pp. 201-209.

Magalhães-Godinho, Vitorino (1969), L'économie de l'empire portugais aux $X V^{e}$ et $X V I^{e}$ siècles, Paris, SEVPEN.

Melis, Federigo (1962), Aspetti della vita economica medievale. Studi nell'archivio Datini di Prato, Florence, L.S. Olschki.

Melis, Federigo (1970), Note sur le mouvement du port de Beyrouth d'après la documentation florentine aux environs de 1400, dans Sociétés et compagnies de commerce en Orient et dans l'Océan Indien. Actes du huitième colloque international d'histoire maritime (Beyrouth-5-10 septembre 1966), Paris, SEVPEN, pp. 371-373.

Melis, Federigo (1972), Documenti per la storia economica dei secoli XIIIXVI, Florence, L.S. Olschki.

Melis, Federigo (1984), Werner Sombart e i problemi nella navigazione nel Medioevo, dans I transporti e le communicazioni nel Medioevo, Florence, Le Monnier, pp. 3-68.

Melis, Federigo (1984), Intensità e regolarità della diffusione dell'informazione economica generale nel Mediterraneo e in Occidente alla fine del Medioevo, dans I transporti e le communicazioni nel Me- 
dioevo con introduzione de Michel Mollat, Florence, Le Monnier, pp. 179-223.

Moukarzel, Pierre (2010), La ville de Beyrouth sous la domination mamelouke (1291-1516) et son commerce avec l'Europe, Baabda, Éditions de l'Université Antonine.

Musso, Giangiacomo (1975), Navigazione e commercio Genovese con il Levante nei documenti dell'archivio di Stato di Genova (secc. XIV-XV), Rome, Pubblicazione degli Archivi de Stato.

Nam, Jank-Kuk (2007), Le commerce du coton en Méditerranée à la fin du Moyen Âge, Leiden, Brill.

Pegolotti, Francesco Balducci (1936), La pratica della mercatura, Cambridge, Medieval Academy of America.

Priuli, Girolamo (1921), I diarii di Girolamo Priuli (1494-1512), dans Rerum Italicarum Scriptores, Raccolta degli storici italiani dal cinquecento al millecinquecento, tomo XXIV-III, volume 1-2-4, Bologna.

Pryor, John H. (2000), Geography, technology and war: Studies in the maritime history of the Mediterranean 649-1571, Cambridge, University Press.

Sardella, Pierre (1948), Nouvelles et spéculations à Venise au début du XVI siècle, Paris, Armand Colin.

Shultz, Warren C. (1995), Mamluk money from Baybars to Barquq: A study based on the literary sources and the numismatic evidence, Chicago, University.

Stöckly, Doris (1995), Le système de l'Incanto des galées du marché à Venise (fin XIII ${ }^{e}$-milieu XV siècle), Leiden, Brill.

Thiriet, Freddy (1978), La crise des trafics vénitiens au Levant dans les premières années du $X V^{e}$ siècle, dans Studi in memoria di Federigo Melis. III, Naples, Giannini, pp. 59-72.

Tucci, Ugo (1958), Sur la pratique vénitienne de la navigation au XVI siècle, "Annales ESC" 13, pp. 72-86.

Vallet, Éric (1999), Marchands vénitiens en Syrie à la fin du XVe siècle: Pour l'honneur et le profit, Paris, Adhe.

Vigouroux, Élodie (2012), La mosquée des Omeyyades de Damas après Tamerlan. Chronique d'une renaissance (1401-1430), "Bulletin d'études orientales", 61, pp. 123-159.

Wake, Christopher (1979), The changing pattern of Europe's pepper and spice imports, ca 1400-1700, "Journal of European Economic History" 8, pp. 361-403.

Fecha de recepción del artículo: enero 2014

Fecha de aceptación y versión final: mayo 2015 